\title{
Correction to: Delayed manifestations of abdominal trauma: follow-up abdominopelvic CT in posttraumatic patients
}

\author{
Khaled Y. Elbanna, ${ }^{1,4}$ Mohammed F. Mohammed, ${ }^{1}$ Shih-Chieh Huang, ${ }^{2}$ David Mak, ${ }^{2}$ \\ J. Philip Dawe, ${ }^{3}$ Emilie Joos, ${ }^{3}$ Heather Wong, ${ }^{3}$ Faisal Khosa, ${ }^{1}$ Savvas Nicolaou ${ }^{1}$ \\ ${ }^{1}$ Emergency \& Trauma Radiology, Vancouver General Hospital, University of British Columbia, Vancouver, Canada \\ ${ }^{2}$ Faculty of Medicine, University of British Columbia, Vancouver, Canada \\ ${ }^{3}$ Trauma Services, Department of Surgery, Vancouver General Hospital, University of British Columbia, Vancouver, Canada \\ ${ }^{4}$ Present address: Toronto, ON, Canada
}

\section{Correction to: Abdom Radiol (2017) https://doi.org/10.1007/s00261-017-1364-4}

The original version of this article unfortunately contained few mistakes. Under the subheading "Data extraction and review process", in line 12 the word "prospective" is incorrectly given by the author. The correct word is "retrospective".
In Fig. 2D, the label should read as RA instead of LA.

In Table 6, the word "ischemic/gangrenous" should read as "ischemia/gangrene" in 9th row, column 6.

The revised fig 2 and table 6 is given below.

The original article can be found online at https://doi.org/10.1007/ s00261-017-1364-4.

Correspondence to: Khaled Y. Elbanna; email: khaledbanna77 (a) gmail.com 

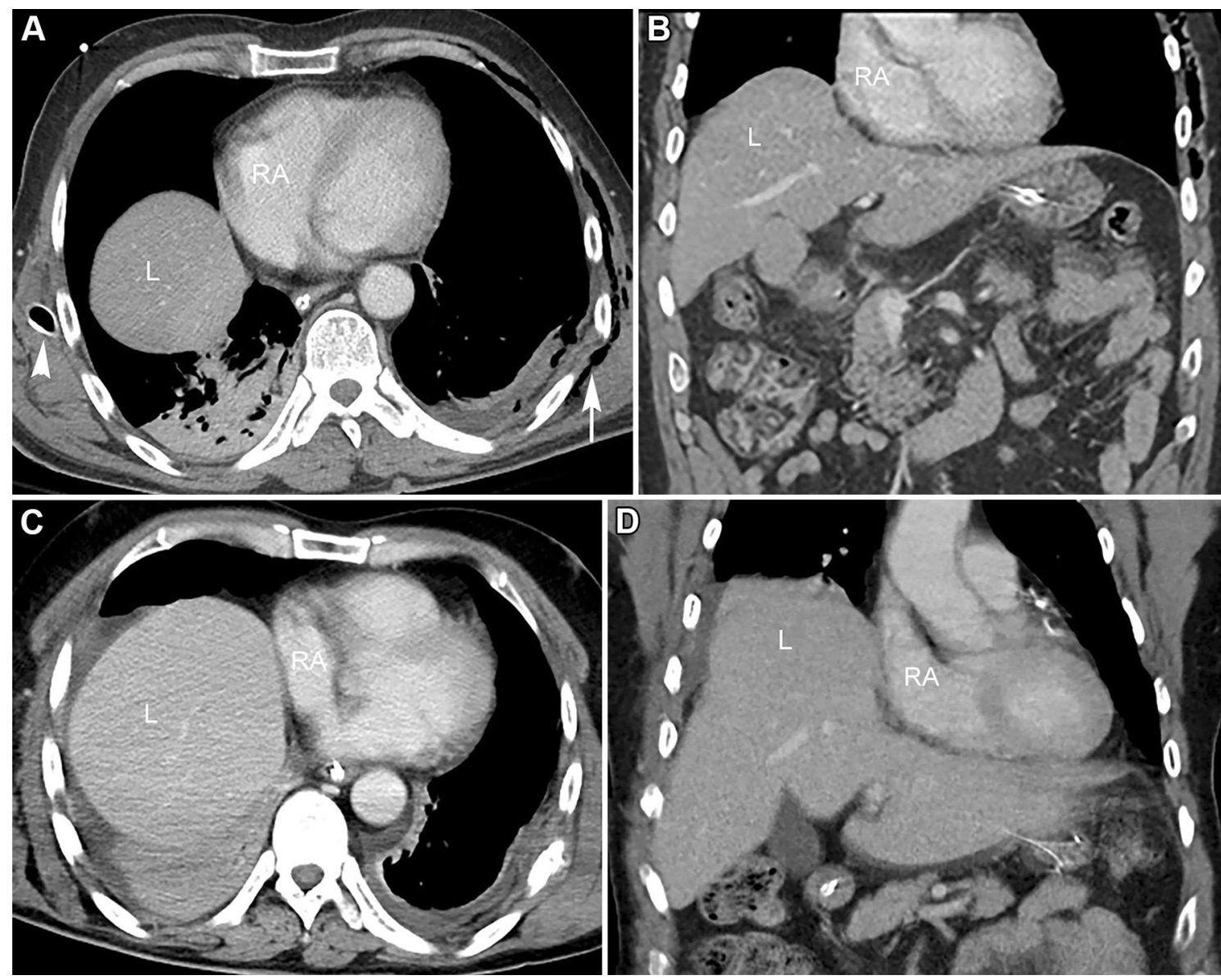

Fig. 2. A 59-year-old man with MVC (thrown from the car on a highway). A and B Axial and coronal reformatted images of IV contrast-enhanced abdominal CT demonstrating an elevation of the right hemidiaphragm with a hump of the right hepatic lobe $(L)$, a finding that was initially missed. Note the relation of the liver to the right atrium (RA). Left rib fracture, subcutaneous gas (arrow), right intercostal tube (arrowhead), and bilateral basal consolidation/atelectasis (right more than

left) are noted. C and D Axial and coronal images of IV contrast-enhanced abdominal CT, 12 days later, demonstrating a more obvious herniation of the liver $(L)$ that is compressing the right atrium (RA) and with a constriction of the liver at the site of the diaphragmatic defect (collar sign). Right basal consolidation/atelectasis, bilateral pleural effusion, and fluid adjacent to the liver dome laterally are also noted.

Table 6. Management of organ-specific findings on follow-up abdominopelvic CT

Organ-specific investigations and management following positive findings on follow-up CT

Spleen

Pancreas

Kidney

Bowel and mesentery

Diaphragm

Miscellaneous

Total Interventions
1 splenectomy

5 coiling/embolization of splenic artery pseudoaneurysms

2 ERCP with subsequent pancreatic duct stenting

1 ureteric stent

4 small bowel surgeries

One patient: SBO due to anastomotic stricture

One patient: traumatic distal ileum perforation

Two patients: traumatic induced small bowel ischemia/gangrene 3 diaphragmatic repair (2 laparotomy +1 laparoscopy)

3 imaging-guided fluid drainage

1 embolization of the left gluteal artery pseudoaneurysm

3 PEG tube replacement

Surgical: 8

Interventional procedures: 15 